\title{
Coincidence experiments between interferometric and resonant bar detectors of gravitational waves
}

\author{
Pia Astone†, $\mathbf{J}$ Alberto Loboł and Bernard F Schutz \\ Department of Physics and Astronomy, University of Wales, College of Cardiff, Cardiff, UK
}

Received 28 February 1994, in final form 13 May 1994

\begin{abstract}
Gravitational wave coincidence experiments between bars and interferometers may be an attractive option once the new generation of full scale interferometers begins taking data. We discuss various ways in which these disparate types of data can be compared in searches for bursts (from supemovae, for example), for pulsar signals, and for a stochastic background. Comparison of broadband interferometer data with narrowband bar data is appropriate in most searches for bursts, but in many cases the results-especially null results (upper limits)-are difficult to interpret. By narrowbanding the interferometer data to the bandwidth of the bar detector, one produces data sets that may give much clearer information in certain burst searches and that are appropriate for searches for a stochastic background of gravitational waves. We suggest, in fact, that there are circumstances where searches for a stochastic background could be more efficiently performed between a bar and an interferometer than between two interferometers. We examine, in some detail, the effect of narrowbanding the interferometer data. We apply this method to a real interferometer and bar data and assess its signal-to-noise performance for different classes of gravitational wave signals.
\end{abstract}

PACS numbers: $0480 \mathrm{~N}, 9555 \mathrm{Y}$

\section{Introduction}

Several groups around the world are developing experiments to search for gravitational waves (see Blair 1991 for a recent comprehensive review). The experiments can be classified into two major categories: those using laser interferometers with very long arms, and those using resonant metallic bars cooled to ultra-low temperatures. The first have the ability to measure the gravitational wave induced strain in a broad frequency band (expected to range from 10 or $100 \mathrm{~Hz}$ up to perhaps $5 \mathrm{kHz}$ ), while the latter measure the gravitational wave Fourier components around the bar's resonant frequency, usually near $1 \mathrm{kHz}$. The new generation of resonant $\mathrm{GW}$ detectors seems to be pointing towards spherical antennæe, with the prospects of increased sensitivity around the first quadrupole modes and being, at the same time, largely omnidirectional.

The two types of detectors are complementary, and it can be useful to obtain information on a possible gravitational wave (GW) from both of them simultaneously. In this paper we consider broadly the kinds of coincident observations that are possible, and the circumstances in which one might choose one or another of them, and we study in detail one method that has not been considered in the literature before, namely filtering the broadband data from

† Permanent address: Istituto Nazionale di Fisica Nucleare, Sezione Università 'La Sapienza', Rome, Italy.

† Permanent address: Departament de Fisica Fonamental, Universitat de Barcelona, Spain. 
an interferometer down to the bandwidth of the bar in order to perform searches for isolated events and for a stochastic background of GWs.

Importantly, there will not be a large number of sensitive GW detectors in operation at any time in the foreseeable future, so one should be ready to compare the data from an interferometer with those obtained with a bar if, for example, the two detectors are in operation during the occurrence of an interesting astrophysical phenomenon, such as a nearby supernova, or if the two detectors are the most sensitive pair for a particular kind of observation. The usual way of quoting the sensitivity of a GW detector is to give its sensitivity to a hypothetical $1 \mathrm{~ms}$ supernova explosion; we call this the detector's 'burst sensitivity'. But for detectors as different as a bar and an interferometer, this is not the whole story, and, in particular, the burst sensitivities do not give a reliable indicator of the sensitivity of the pair of detectors for all kinds of observations.

In the next section we catalogue the different kinds of observations that a bar and an interferometer might perform together, and we show that one can deduce their relative sensitivities if one knows not only the burst sensitivity of each detector but also the bar's bandwidth. By adopting simple models of the detectors and the expected signal waveforms, we assess the usefulness of comparisons of broadband data with narrowband data and of narrowbanding the interferometer data before performing a comparision.

We then discuss, in detail, the case of narrowbanding the interferometer data, based on more detailed and realistic models of a bar and an interferometer. We do this by adding simulated signals to real data from the Garching detector (Maischberger et al 1991), then narrowbanding to the characteristics of the Rome bar detector NAUTLUS.

\section{Options for coincidence experiments between bars and interferometers}

In this section we survey ways in which bars and interferometers can perform coincident observations, and we make rough estimates of the sensitivity of each method, in order to assess the conditions under which it might be appropriate to use the method. We consider three principal types of GWs; short bursts with little predictable structure, continuous signals of fixed frequency, and a stochastic background of radiation left over from the big bang. We consider observations of these waveforms using combinations of three possible detectors: a resonant bar detector, a broadband interferometer, and an interferometer that has been made narrowband by an appropriate choice of optical configuration, such as resonant recycling (Drever 1983) or dual recycling (Meers 1988).

\subsection{Simple wave models}

Because we want to keep our estimates simple, we adopt crude models of our three sources:

(i) A short burst is a featureless waveform that rises quickly to an amplitude $h_{\mathrm{b}}$ and decays to zero just as quickly after a time $\tau_{b}$. Its Fourier transform $\tilde{h}$ is equally featureless, with an amplitude $h_{\mathrm{b}} \tau_{\mathrm{b}}$ and a bandwidth extending from zero frequency to $1 / \tau_{\mathrm{b}}$. We can summarize as follows.

Short burst:

amplitude $h_{\mathrm{b}}$, duration $\tau_{\mathrm{b}}$

transform amplitude $h_{\mathrm{b}} \tau_{\mathrm{b}}$, bandwidth $1 / \tau_{\mathrm{b}}$. 
(ii) A continuous wave of amplitude $h_{\mathrm{c}}$ and frequency $f_{\mathrm{c}}$ that is constant for the entire observation time $T_{\text {obs }}$ has a similarly rough description.

Continuous wave:

$$
\begin{aligned}
& h(t)=h_{\mathrm{c}} \cos \left(2 \pi f_{\mathrm{c}} t\right) \\
& \text { transform amplitude } \frac{1}{2} h_{\mathrm{c}} T_{\text {obs }} \\
& \text { bandwidth } 1 / T_{\text {obs }}, \text { about } f= \pm f_{\mathrm{c}} .
\end{aligned}
$$

(iii) A stochastic background is a random $h(t)$ that has an RMS amplitude $h_{5}$. We assume its spectrum is flat and that its energy density per unit logarithmic frequency is a fraction $\Omega_{\mathrm{gw}}(f)$ of the closure density $\rho_{\mathrm{c}}$ of the universe:

$$
\frac{\mathrm{d} \rho_{\mathrm{gw}}}{\mathrm{d} \ln f}=\Omega_{\mathrm{gw}} \rho_{\mathrm{c}} \text {. }
$$

In these terms the background may be characterized by the power spectral density of any single metric component in TT gauge. We call this $S_{\mathrm{gw}}(f)$, and it is given by

$$
S_{\mathrm{gw}}(f)=\frac{2 G}{\pi} f^{-3} \Omega_{\mathrm{gw}}(f) \rho_{\mathrm{c}}
$$

\subsection{Simple detector models}

Any present and planned gravitational wave detector can be described as a linear measuring instrument whose output is a data stream $x(t)$ containing the $\mathrm{GW}$ signal $h(t)$ and additive noise $n(t) \dagger$. Different detectors are distinguished from one another by having different noise characteristics, characterized by the (two-sided) power spectral density of noise $S_{\mathrm{h}}(f)$

$$
S_{\mathrm{h}}(f)=\int_{-\infty}^{\infty}\left\langle n\left(t^{\prime}\right) n\left(t^{\prime}+t\right)\right\rangle \mathrm{e}^{-2 \pi \mathrm{i} f t} \mathrm{~d} t
$$

where $\left\{n\left(t^{\prime}\right) n\left(t^{\prime}+t\right)\right\}$ is the autocorrelation of the noise in the signal output stream, which is assumed independent of $t^{\prime}$ (stationary noise).

The optimum performance of a detector searching for a signal of known waveform $h(t)$ contained in the output stream is obtained by filtering the output with a filter matched to the signal. The amplitude signal-to-noise ratio $S / N$ of the output of the filter is given by the well known formula

$$
\left(\frac{S}{N}\right)^{2}=\int_{-\infty}^{\infty} \frac{|\tilde{h}(f)|^{2}}{S_{\mathrm{h}}(f)} \mathrm{d} f .
$$

Although the integration range is infinite in theory, in practice it will be reduced to the Nyquist band for any specified set of sampled data. For a sampling interval $\Delta t$, the Nyquist band is $(-1 / 2 \Delta t, 1 / 2 \Delta t)$.

The two detector models we consider are as follows.

(i) A broadband interferometric detector has a flat noise spectrum $S_{\text {int }}$ over any interesting signal bandwidth. It is common to quote the sensitivity of such a detector by giving the amplitude of a $1 \mathrm{~ms}$ gravitational wave burst that would have a signal-to-noise ratio of 1 if the data stream were filtered with a filter matched to the signal. For the broadband burst and our simple interferometric detector model, (6) gives 1 when

$$
S_{\text {int }}=2 h_{\mathrm{b}}^{2} \tau_{\mathrm{b}} .
$$

$\dagger$ Instrumental transfer functions have to be removed from actual output streams to get back to $h(t)$. This is the usual way that interferometric detectors are described, but readers should be aware that this is not the way bar detectors are usually described in the literature. 
Therefore an interferometer of sensitivity $h_{b}$ for millisecond bursts has a noise spectrum given by

BB interferometer: $S_{\mathrm{h}}(f)=S_{\text {int }}=2 \times 10^{-3} h_{\mathrm{b}}^{2} \mathrm{~Hz}^{-1} \quad 0 \leqslant|f|<1 / \Delta t_{\text {int }}$

where $\Delta t_{\text {int }}$ is the sampling interval for the interferometer, assumed much shorter than the signal duration of $1 \mathrm{~ms}$. We do not distinguish between ordinary and recycling interferometers. These are sufficiently similar for our purposes in this section.

(ii) A bar detector is intrinsically narrowband. It may be crudely described by taking its sensitivity to be confined to a bandwidth $B_{\mathrm{b}} \dagger$ about a frequency $f_{0}$; we approximate $S_{\mathrm{h}}(f)$ by a constant $S_{\mathrm{b}}$ in this region and by infinity outside this band.

Present bars have rather narrow bandwidths, of the order of $2 \mathrm{~Hz}$ (Astone et al 1993). Note that this is not the width of the resonance of the bar, which is much narrower. Rather, it is set by the noise in the signal readout system: if this were noise-free, so that arbitrarily small excitations of the bar could be measured, then the bar could be used to detect signals that are well off-resonance. We give a fuller discussion of bar detectors in section (3) below.

However, bars are not usually described by giving their noise spectral density. Rather, their sensitivity is quoted in the same way as are broadband interferometers, by giving the amplitude of a broadband burst of $1 \mathrm{~ms}$ duration which would register a $S / N$ of 1 in a matched filter. Using (6) as before, but recognizing that the detector registers the signal only in its bandwidth $B_{b}$, gives

$$
S_{\mathrm{b}}=2 h_{\mathrm{b}}^{2} \tau_{\mathrm{b}}^{2} B_{\mathrm{b}} .
$$

This leads to

bar detector: $\quad S_{\mathrm{b}}(f)= \begin{cases}4 \times 10^{-6} h_{\mathrm{b}}^{2}\left(B_{\mathrm{b}} / 2 \mathrm{~Hz}\right) \mathrm{Hz}^{-1} & \text { if }\left|f-f_{\mathrm{b}}\right|<B_{\mathrm{b}} / 2 \\ \infty & \text { otherwise. }\end{cases}$

For our purposes, a bar is then completely described by giving two numbers: its sensitivity $h_{\mathrm{b}}$ to millisecond bursts and its bandwidth $B_{\mathrm{b}}$.

It is clear that the sensitivity of a detector depends on the model adopted for the signal, and that an interferometer and a bar that are nominally of similar sensitivity for broadband bursts can have very different sensitivities for other signals. The bar will be much more sensitive than the comparable interferometer within the bar's bandwidth, and it will be much less sensitive than the interferometer outside that band.

We note, in passing, that it is possible to convert an interferometer into a narrowband instrument by physically changing the optics (Drever 1983, Meers 1988). If this is done then the interferometer behaves for our purposes much like a bar detector, although the bandwidth would be rather larger than present bars can achieve. If physically narrowbanding the interferometer makes its sensitivity similar to that of a bar, then this might be an attractive option for a dedicated search, but the treatment of this intrinsically narrowband data is not the subject of this paper. Here we restrict our considerations to interferometer data that is intrinsically broadband, but which one might want to filter down to the bar's bandwidth after it has been acquired.

$\dagger B_{\mathrm{b}}$ is related to the parameter $\beta_{3}$ of the bar that we introduce in sections 3 and 5 by $B_{\mathrm{b}}=2 \beta_{3} / 2 \pi$. 


\subsection{Types of coincidence experiments}

In this section we describe various ways of performing coincidence experiments between bars and interferometers, and we discuss what modes might be appropriate for detectors of various relative sensitivities.

\subsubsection{Searching for bursts with bars and interferometers. A detector optimizes its} sensitivity to a given signal by making its bandwidth as large as possible, up to the signal bandwidth. If we are searching for millisecond bursts by comparing the output of a bar and a broadband interferometer, the most sensitive search mode is to filter both optimally. This means comparing $1 \mathrm{kHz}$ bandwidth interferometer data with narrowband bar data. However, in certain circumstances, such as when the interferometer's broadband sensitivity is much greater than that of the bar, it may also make sense to filter the interferometer data down to the bar's bandwidth.

When the burst sensitivities of the bar and the broadband interferometer are similar (a 'balanced' coincidence experiment), then comparison of the broadband interferometer data with the bar output is the only feasible approach. The interferometer data are sampled at, say, $20 \mathrm{kHz}$, while the bar data will be sampled at, say, $55 \mathrm{~Hz}$.

There is a serious difficulty with such a search. The different observing bandwidths of the two detectors mean that their data streams contain very different information. Real burst signals will not have perfectly flat spectra up to $1 \mathrm{kHz}$, and so it is possible for a burst to register in the interferometer and not in the bar, or to excite the bar relatively more strongly than the interferometer. In particular, this means that a negative outcome to a coincidence experiment must be interpreted with care: it does not mean that bursts of the nominal detector sensitivity were not present, but rather that only bursts at that amplitude that also had very flat spectra were absentt. If a coincidence is observed, then unless it has a very strong signal-to-noise ratio, the interferometer will not be able to give spectral information in the bar's bandwidth. It will not be possible, for example, to use the antenna patterns of the detectors to give reliable constraints on the direction of the source.

If the sensitivities of the two detectors are not 'balanced', then the best strategy may be different. If the bar has a burst sensitivity significantly better than that of the interferometer, then there is little that can be done. A coincidence search can be made at the sensitivity of the interferometer, using broadband interferometer data as above. Any events that are found would have been recognized as very unusual even if the bar had been operating alone, but the interferometer in this case provides the confirmation that the event was not caused by unmodelled noise in the bar.

On the other hand, if the interferometer has a burst sensitivity much greater than that of the bar, it becomes attractive to use only the interferometer data within the bar's bandwidth to perform a much tighter coincidence analysis, in addition to the broadband search described above.

A broadband interferometer will have the same sensitivity as a bar within the bar's bandwidth if it has the same spectral noise density, i.e. if $S_{\text {int }}=S_{\mathrm{b}}$. By using our previous expressions for the noise in these detectors, it follows that the burst sensitivity of the interferometer must be better than that of the bar by a factor

$$
\frac{h_{\mathrm{b}, \text { int }}}{h_{\mathrm{b}, \mathrm{bar}}}=0.05\left(\frac{B_{\mathrm{b}}}{2 \mathrm{~Hz}}\right)^{1 / 2} \text {. }
$$

† This problem also exists for coincidence experiments among different bar detectors whose observing frequencies differ from one another. 
If this is realized, the greater broadband sensitivity of the interferometer would mean that any coincident event would probably have been recognized as unusual in the interferometer data alone, and the bar would provide the confirmation that it was real. But in addition, by particularly using the spectral information in the bar's bandwidth, the experiment can compare like with like. This has at least three attractive uses.

(i) If a coincident event is identified between broadband interferometer data and the bar data, then one would expect the event to be coincident in the narrowbanded interferometer data as well. This provides further discrimination against spurious coincidences.

(ii) A coincidence in the bar's bandwidth allows antenna beam patterns to place more reliable constraints on the position of the source.

(iii) Perhaps the most important use of narrowbanding the interferometer data is for searches that turn up no coincidences in the bar's bandwidth. In this case one can infer secure and clear-cut upper limits on broadband bursts that have significant power at the bar's frequency.

We study the implementation of a narrowbanded analysis in some detail below.

2.3.2. Searching for continuous signals with bars and interferometers. A continuous signal can only be seen by the bar if it is within the bandwidth of the bar. For either instrument, the signal-to-noise ratio will be given by

$$
\frac{S}{N}=\frac{h_{\mathrm{c}}}{\sqrt{2 S_{\mathrm{b}} / T_{\text {obs }}}} .
$$

If $T_{\text {obs }}$ is long enough, this sensitivity will only be achieved if the output is filtered to remove the Doppler shifts in the incoming signal that are produced by the motion of the Earth. This also serves to increase confidence in the observation of a signal by a single detector, since it would seem to be unlikely that external interference could produce a signal that had the same pattern of Doppler shifts. Nevertheless, confirmation of an observation in two detectors would be very useful.

The ratio of sensitivities of the two detectors to a continuous signal in the bar's bandwidth is therefore the inverse ratio of the square roots of their power spectral densities:

$$
\frac{h_{\mathrm{c}, \text { int }}}{h_{\mathrm{c}, \mathrm{bur}}} \simeq 25 \frac{h_{\mathrm{b}, \text { int }}}{h_{\mathrm{b}, \mathrm{bar}}}\left(\frac{B_{\mathrm{b}}}{2 \mathrm{~Hz}}\right)^{-1 / 2} .
$$

So if the bar and interferometer have similar burst sensitivities $\left(h_{\mathrm{b}, \text { int }}=h_{\mathrm{b}, \mathrm{bar}}\right)$, then the bar will be roughly 25 times more sensitive to continuous signals in its bandwidth. Alternatively, an interferometer whose burst sensitivity is 25 times that of a bar could search for continuous signals over its entire bandwidth with the same sensitivity that the bar has in its narrow band $\dagger$.

2.3.3. Stochastic signals. We shall make the usual assumption that a stochastic background of gravitational radiation is a stationary noise process. This means that the random signal in any frequency band of width $1 / T_{\text {obs }}$ (the frequency resolution in the experiment) is uncorrelated with the signal in any other band. It follows that two different detectors can only respond to the background in a correlated way if they look at signals in the same

† A more rigorous and realistic treatment (see Pallottino and Pizzella 1984) shows that a numerical factor is actually present in (13). Its value is around 2 for the EXPLORER antenna. 
bandwidth†. It follows that broadband interferometer data must be filtered to the bar's bandwidth before performing a cross-correlation search for a stochastic background.

The signal-to-noise ratio of a GW background in a cross-correlation experiment between two detectors located near one another is, using notation developed above (cf Schutz 1991)

$$
\frac{S}{N}=\left(\frac{S_{\mathrm{gw}}^{2}}{S_{\mathrm{int}} S_{\mathrm{b}}} B_{\mathrm{b}} T_{\mathrm{obs}}\right)^{1 / 4} \text {. }
$$

Detectors that are separated by some distance are not as well correlated, because radiation coming from within a certain cone about the line joining the detectors will reach one detector well before the other. The fall-off in the correlation with separation is a function of the ratio of the wavelength to the separation, and it has been studied for pairs of bars and pairs of interferometers (Michelson 1987), a forthcoming paper will study it in detail for bar-interferometer correlations (Compton et al 1993).

Supposing the detectors are immediately adjacent and aligned for optimum correlation, the background will reach a signal-to-noise ratio of 1 if its fraction of the cosmological closure density is

$$
\Omega_{\mathrm{gw}}=1.5 \times 10^{-5}\left(\frac{f}{10^{3} \mathrm{~Hz}}\right)^{3}\left(\frac{h_{\mathrm{int}}}{10^{-21}}\right)\left(\frac{h_{\mathrm{b}}}{10^{-21}}\right)\left(\frac{T_{\mathrm{obs}}}{10^{7} \mathrm{~s}}\right)^{-1 / 2} .
$$

where we have assumed the Hubble constant to be $100 \mathrm{~km} \mathrm{~s}^{-1} \mathrm{Mpc}$. The next generation of bars and interferometers can therefore begin setting interesting limits on the background at kilohertz frequencies.

This equation reveals an important feature of cross-correlation experiments: the sensitivity depends only on the burst sensitivity of the bar, not on its bandwidth. In particular, a 'bar' whose bandwidth is $1 \mathrm{kHz}$-in other words, an interferometer-could be substituted for the narrowband bar and the experiment would have exactly the same stochastic sensitivity, provided the new interferometer's burst sensitivity were the same as that of the narrowband bar. Viewed another way, this means that a bar-interferometer correlation would be just as sensitive as an interferometer-interferometer correlation at $1 \mathrm{kHz}$, provided that all the detectors had the same burst sensitivity.

The next generation of large-scale interferometers will be separated by distances much larger than the wavelength of gravitational waves at $1 \mathrm{kHz}$. If the separation reduces the sensitivity of a two-interferometer search by, say, a factor of 10 , then a correlation between one of the interferometers and a nearby bar whose burst sensitivity is worse by a factor of 10 would still have the same sensitivity. We therefore draw the important conclusion that, it may be worthwhile moving ultra-cryogenic bars very near to single interferometers to perform stochastic searches near $1 \mathrm{kHz}$, especially when the interferometers are at their first stage of sensitivity $\left(h_{\mathrm{b}} \sim 10^{-21}\right)$. The new NAUTILUS bar in Rome (see below) and the VIRGO interferometer planned for Pisa (Bradaschia et al 1990) are only a little too far apart for an optimum correlation at the frequency of the bar.

2.3.4. Summing up. There are situations in which it would be useful to perform joint searches for gravitational waves using bars and interferometers. Searches for bursts using the full bandwidths of both detectors will always be interesting, and the estimates we have provided here are probably sufficient to give a good idea of the sensitivity of such searches.

$\dagger$ We ignore the possibility that one detector is moving with respect to the other, or that one accelerates by a significant amount during the time it takes a wave to travel between the detectors. For ground-based detectors, these effects are negligible. 
Where it is appropriate to narrowband the interferometer data by filtering it to the bar's bandwidth, such as for a stochastic search or for certain burst searches, the results we have derived here may be a little crude, since our model of the bar is rather crude. Therefore, in the rest of this paper we study narrowbanding experiments in more detail, including an analysis of the effect of narrowbanding on real interferometer data.

\section{Model of the resonant bar detector}

The bar detector is, typically, a metallic cylindrical bar, usually of high quality aluminium alloy. The mass of the bar needs to be as large as possible in order to capture as much energy as possible from the impinging gravitational wave (GW). When the bar is hit by a GW it starts to vibrate at those resonance modes that are coupled to the GW. The vibrations of the bar are usually observed at its first longitudinal mode, with frequency $f_{0} \sim v / 2 L$, where $v=5400 \mathrm{~m} \mathrm{~s}^{-1}$ is the velocity of sound in aluminium, and $L$ is the bar's length.

The EXPLORER detector of the Rome group (Astone et al 1993) is a $3 \mathrm{~m}$ long bar, weighing about $2300 \mathrm{~kg}$, and with $f_{0}$ about $900 \mathrm{~Hz}$. The vibration $\xi(t)$ of the bar's end face is converted into an electrical signal $V(t)$ by an electromechanical transducer with constant $\alpha=V(t) / \xi(t)$.

The electrical signal from the transducer is amplified with a wideband very low noise preamplifier, and then processed by means of optimum filters in order to make the signalto-noise ratio (SNR) as large as possible. It can be shown that if the bar is hit by a GW burst then the vibration amplitude $\xi(t)$ is

$\xi(t)=\frac{4 f_{0} L}{\pi} \mathrm{e}^{-t / \tau_{v}} \tilde{h}\left(f_{0}\right) \sin \left(2 \pi f_{0} t\right) \sin ^{2} \theta \cos 2 \phi=\xi_{0} \mathrm{e}^{-t / \tau_{\nu}} \sin \left(2 \pi f_{0} t\right)$

where $\tilde{h}\left(f_{0}\right)$ is the Fourier component of the burst at frequency $f_{0}, \tau_{v}=Q / \pi f_{0}=1 / \beta_{1}$ is the relaxation time ( $Q$ is the merit factor), $\theta$ is the angle between the incoming wave and the bar axis and $\phi$ is the angle between the wave polarization plane and the bar axis. The total energy associated with the vibration is

$$
E=\pi^{2} M f^{2} \xi_{0}^{2} \text {. }
$$

If the wave arrives perpendicularly to the bar axis and has optimum polarization-i.e. $\theta=\pi / 2, \phi=0$-we can derive

$$
h_{\mathrm{b}}=\frac{1}{\tau_{\mathrm{b}}} \frac{L}{v^{2}}\left(\frac{E}{M}\right)^{1 / 2}
$$

in the approximation $\tilde{h}\left(f_{0}\right) \simeq h_{\mathrm{b}} \tau_{\mathrm{b}}$, where $h_{\mathrm{b}}$ is the burst amplitude and $\tau_{\mathrm{b}}$ its short duration.

As we have stressed earlier, (18) is based on a particular assumption about the wave spectrum, because the bar detects only the spectral components of $h(t)$ near its resonance frequency.

For $M=2300 \mathrm{~kg}, L=3 \mathrm{~m}, \tau_{\mathrm{b}}=1 \mathrm{~ms}$, we have

$$
h_{\mathrm{b}}=8 \times 10^{-18} \mathrm{E}^{1 / 2}
$$

where the energy $E$ is expressed in Kelvin in order to compare signals and noise of the two detectors.

As regards the noise, we have two basic sources: the first comes from the thermal motions of the bar constituents (Brownian noise) and the second is due to the electronic preamplifier (electronic noise). The latter has a spectrum $S_{0}$, which can be assumed white in the frequency range of observation. The preamplifier noise also heats the bar with a 


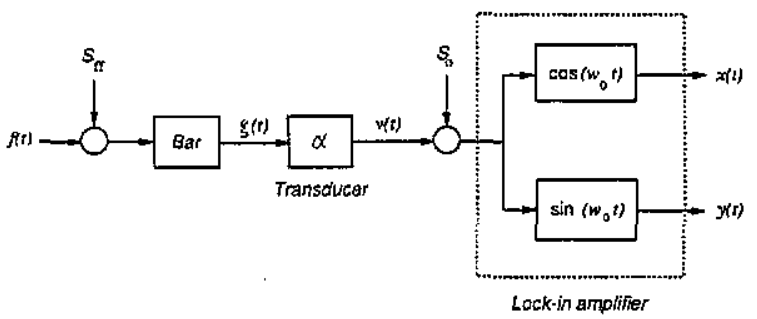

Figure 1. Block diagram of antenna, transducer and lock-in amplifier. $S_{0}=$ white noise spectrum, $S_{\mathrm{ff}}=$ Brownian noise plus back-action noise spectrum.

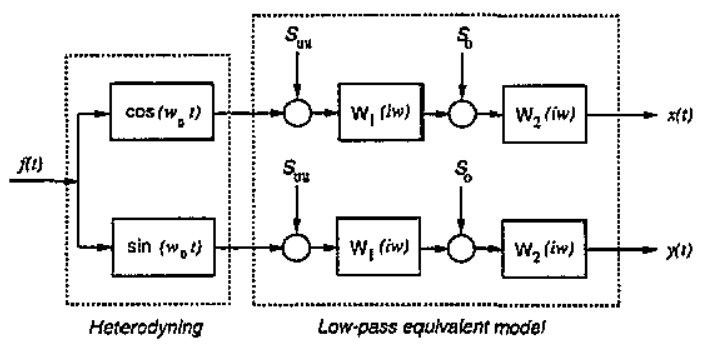

Figure 2. Block diagram of the low pass equivalent model. $S_{0}=$ white noise spectrum, $S_{\mathrm{uu}}$ $=$ narrowband noise spectrum at the bar resonance frequency, $W_{1}=$ transfer function of the mechanical oscillator representing the bar, $W_{2}=$ transfer function for the filtering action of the lock-in.

back-action force which increases the Brownian noise to an equivalent temperature level $T_{\mathrm{e}}$.

The bar detector has actually more than one resonance frequency. When using a resonant electromechanical transducer it can be shown that there are two resonance frequencies

$$
f_{1,2}=f_{0}\left(1 \pm \frac{1}{2} \mu^{1 / 2}\right)
$$

where $\mu(\ll 1)$ is the ratio of the transducer and the bar masses. Typically, the separation between the two modes is of the order of $15 \mathrm{~Hz}$. This means that the transducer output can be processed with two lock-in amplifiers driven at the frequencies of the two modes, or it can also be directly sampled. In what follows we will refer to data acquisition by means of two lock-in amplifiers. With this configuration the two resonant modes are observed and filtered separately. The lock-in amplifier translates the observations from $f_{1}\left(f_{2}\right)$ to $\mathrm{DC}$. Therefore the data can be sampled at a relatively slow rate, related to their bandwidth rather than to the frequency $f_{1}\left(f_{2}\right)$. The two output signals of the lock-in are proportional to the real and imaginary parts of the complex oscillation of the observed antenna mode.

The block diagram of the antenna, tranducer and lock-in amplifier is shown for one of the two modes in figure 1 . The diagram is the same for the second mode. It can be shown (Bonifazi et al 1978) that the system of figure 1 can be represented by the equivalent model given in figure 2, where $S_{\mathrm{uu}}$ is the narrowband noise spectrum at the resonance frequency and $S_{0}$ is the wideband noise spectrum observed sufficiently far from the two resonance frequencies. Here we also have that $W_{1}=\beta_{1} /\left(\beta_{1}+\mathrm{i} \omega\right)$ represents the transfer function of the mechanical oscillator, as seen through the lock-in amplifier, and $W_{2}=\beta_{2} /\left(\beta_{2}+\mathrm{i} \omega\right)$ represents the filtering action of the lock-in amplifier.

In the absence of signals and of non-Gaussian disturbances the two outputs of the lock- 
in are Gaussian variables (the noise, both thermal and electronic, has Gaussian distribution with zero mean). If $x(t)$ and $y(t)$ are the two output components of the lock-in, we usually consider the quantity

$$
r(t)=\left[x^{2}(t)+y^{2}(t)\right]^{1 / 2}
$$

whose square represents the energy of the antenna's resonant mode. The variable $r^{2}(t)$ has exponential distribution with mean value $T_{e}$. The overall sensitivity of the apparatus for short burst detection, obtained by means of optimum filters, is usually expressed by the effective noise temperature

$$
T_{\text {eff }} \simeq 4 T_{\mathrm{e}} \sqrt{\Gamma}
$$

where $\Gamma=S_{0} / S_{\mathrm{uu}}$ is the spectral ratio, typically $\ll 1$.

The best estimation of an input signal of short duration acting on the antenna can be obtained by using the theory of the optimum filter of Wiener-Kolmogorov. The estimation of the two components of the input signal is obtained by filtering each of the two streams of the observed data $x(t)$ and $y(t)$ with the transfer function (Astone et al 1991)

$$
W(\mathrm{i} \omega)=\frac{1}{1+\Gamma} \frac{1}{W_{1} W_{2}} \frac{\beta_{3}^{2}}{\beta_{3}^{2}+\omega^{2}}
$$

where $\beta_{3} \simeq \beta_{1} / \Gamma^{1 / 2}$ defines the overall bandwidth of the detection system (i.e. including the filter). This bandwidth is much larger than the mechanical bandwidth of the antenna oscillator $\left(\beta_{3} \gg \beta_{1}\right)$, as can be simply understood noticing that the bar responds in the same way to an excitation due to the GW and to the Brownian noise and, therefore, its bandwidth is limited only by the noise of the electronic amplifier.

In the filter transfer function $1 / W_{1} W_{2}$ is an inverse filter that cancels the dynamics of the antenna, and $\beta_{3}^{2} /\left(\beta_{3}^{2}+\omega^{2}\right)$ is a non-causal smoothing filter, that minimizes the contribution of the wideband noise.

\section{The interferometric detector}

The interferometric gravitational wave detector is a Michelson or Fabry-Perot interferometer which is capable of sensing the phase changes in a laser beam that are induced by an incoming GW (Giazotto 1989, Lobo 1992). Large-scale detectors of this kind will soon be constructed: the LIGO project (Abramovici et al 1991) and the VIRGO project (Bradaschia et al 1990). There are at present a few smaller scale prototypes capable of achieving burst sensitivities near $10^{-18}$ which are being used to develop techniques that the larger versions will require.

During March 1989 two of these prototypes-at Glasgow (Ward et al 1991) and at Garching, near Munich (Maischberger et al 1991)—took data in parallel for a period of 100 hours. Although it was not likely that real GW signals would be seen during an experiment of this sensitivity, the experiment proved to be very useful in demonstrating that such detectors can operate over sustained periods of time, their duty cycles being as high as $99 \%$ and $89 \%$ for Garching and Glasgow, respectively. The run also provided valuable information about several other matters, such as data handling problems, long term noise control, and so on, and, very importantly, the 100 hour data have been used to test a variety of data analysis techniques, both on each set separately (Watkins 1991 and Niebauer et al 1993), and in coincidence between the two antenne (papers in preparation). We shall use some of this data in our analysis below. 


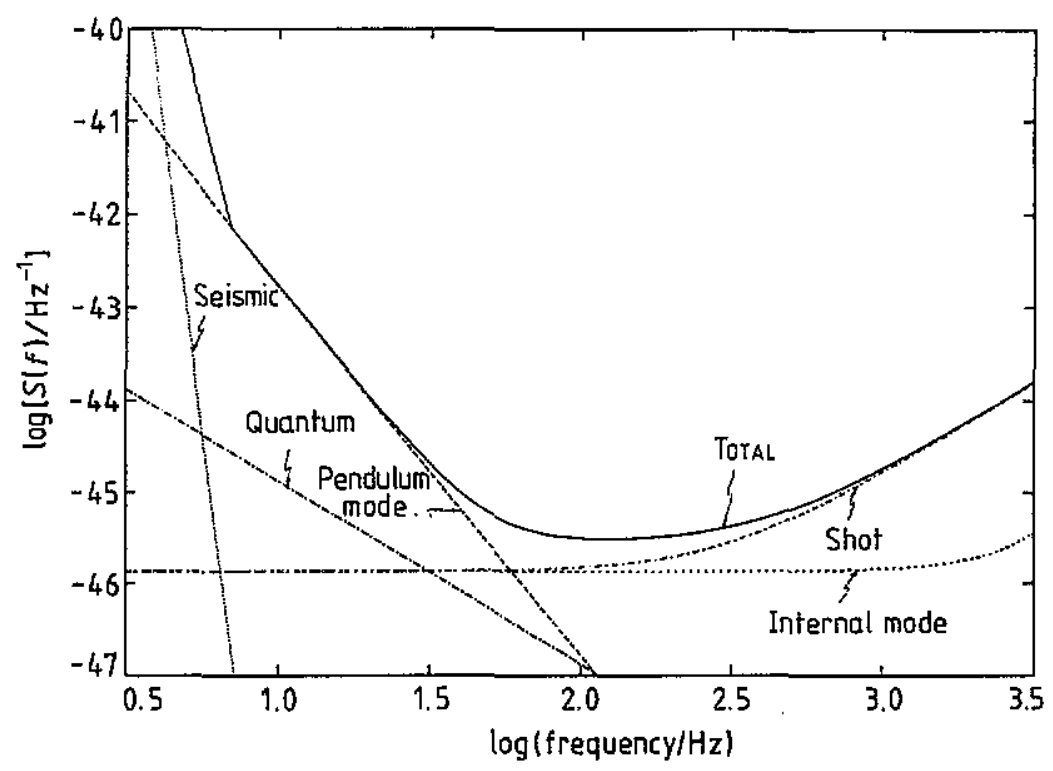

Figure 3. Estimate of the different sources of noise expected to contaminate the future LrGo interferometric detectors.

Interferometric detectors are intrinsically broadband, so data sampling rates are much higher than in resonant bars. Glasgow took data at $20 \mathrm{kHz}$, and Garching at $10 \mathrm{kHz}$-thus spanning $10 \mathrm{kHz}$ and $5 \mathrm{kHz}$ spectral ranges, respectively. Future full scale devices will have bandwidths in this range, too.

Noise in such detectors comes from a variety of different sources, each of them being dominant in different regions of the frequency spectrum: seismic, thermal and man-made noise will be the most serious sources at low frequencies, whereas shot noise (due to photoncounting fluctuations in the photodetector) is likely to be the main concern in the higher part of the spectrum. Figure 3 shows a plot of the contributions of several varieties of noise - the ones believed to be most relevant-to the total noise. The data are taken from Finn and Chernoff (1993), and they constitute an estimate of the likely performance of a realistic full scale detector.

Figure 4 shows a plot of the noise spectral density of the Garching prototype, estimated from a short stretch of data (about one minute long), excerpted from the 1989100 hour run. This noise is of course larger than will be expected in the future antenna. It has many narrow spikes of randomly varying width, roughly between 5 and $20 \mathrm{~Hz}$. These spikes are caused by mains noise-and this is the reason why they happen every $50 \mathrm{~Hz}$. Hopefully, such spikes will be removed in future interferometer designs by use of suitable filtering devices.

The sensitivity to a $1 \mathrm{~ms}$ burst during the analysed stretch of time is

$$
h_{\text {Garching }}=3.7 \times 10^{-17}
$$

which is fairly representative of the entire 100 hour set (Nicholson, private communication). As mentioned earlier, this stretch of data has been utilized in a part of our analysis to be described below. 

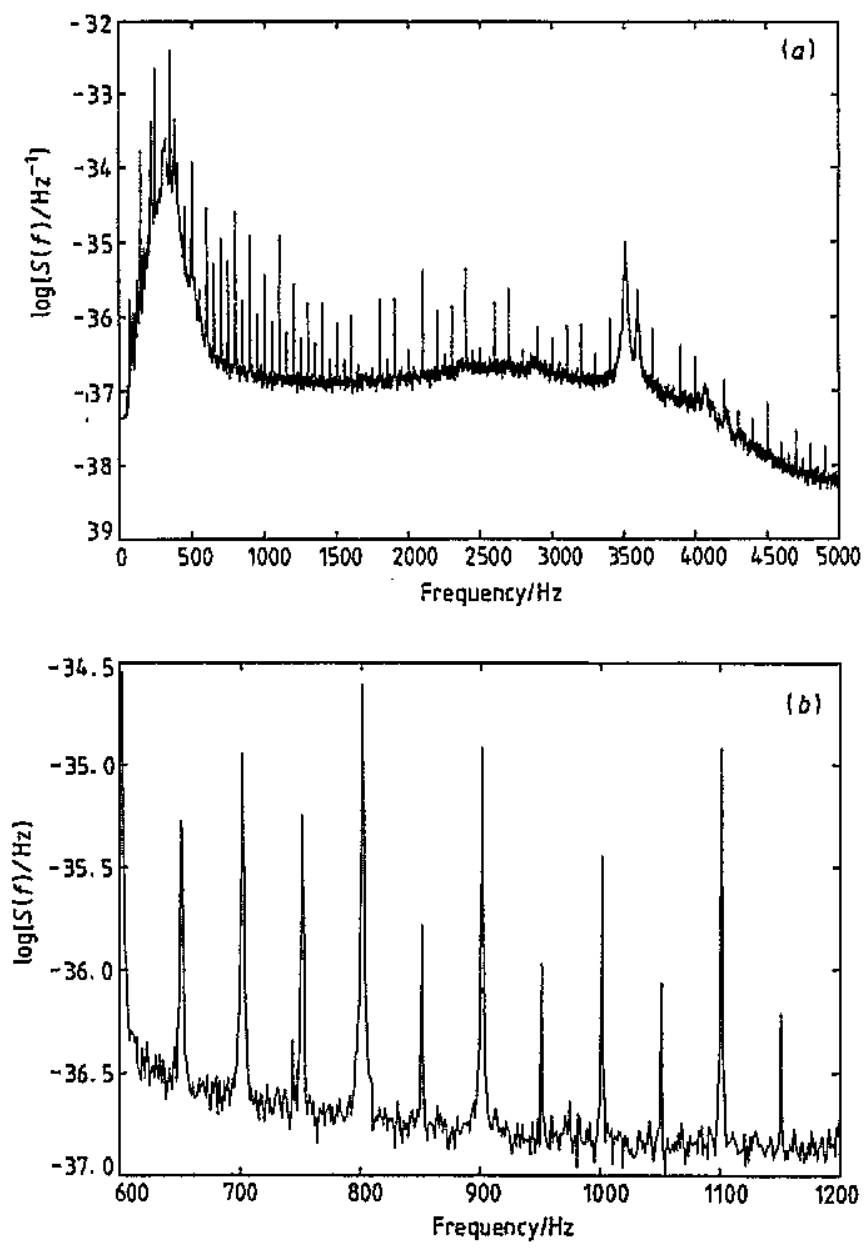

Figure 4. (a) Estimated noise spectrum of the Garching interferometer. (b) Close up of part (a).

\section{Narrowbanding of the interferometer data}

As we discussed earlier, the difference in bandwidths of a bar and an interferometer make comparison of their outputs difficult. We discuss here the approach of narrowbanding the interferometer data down to the bandwidth of the bar. One can then apply to the output of this filtering procedure (or chain, as we shall often call it) and to the bar data identical coincidence criteria, chosen suitably for a given experiment. This procedure removes all the ambiguities associated to the qualitative differences in performance of the two kinds of detectors, but of course in searches for bursts it generally results in a reduction in the SNR for the interferometer data. We estimate this effect here.

A convenient way to represent the narrowbanding process is to look upon the interferometer data as if they were a real background of gravitational radiation impinging on the resonant bar, which is in its turn modelled as a chain of filters that models the response of the bar to this background. Figure 5, right, shows the block diagram of the chain.

First, the data are separately heterodyned at the two bar resonance frequencies, in order 


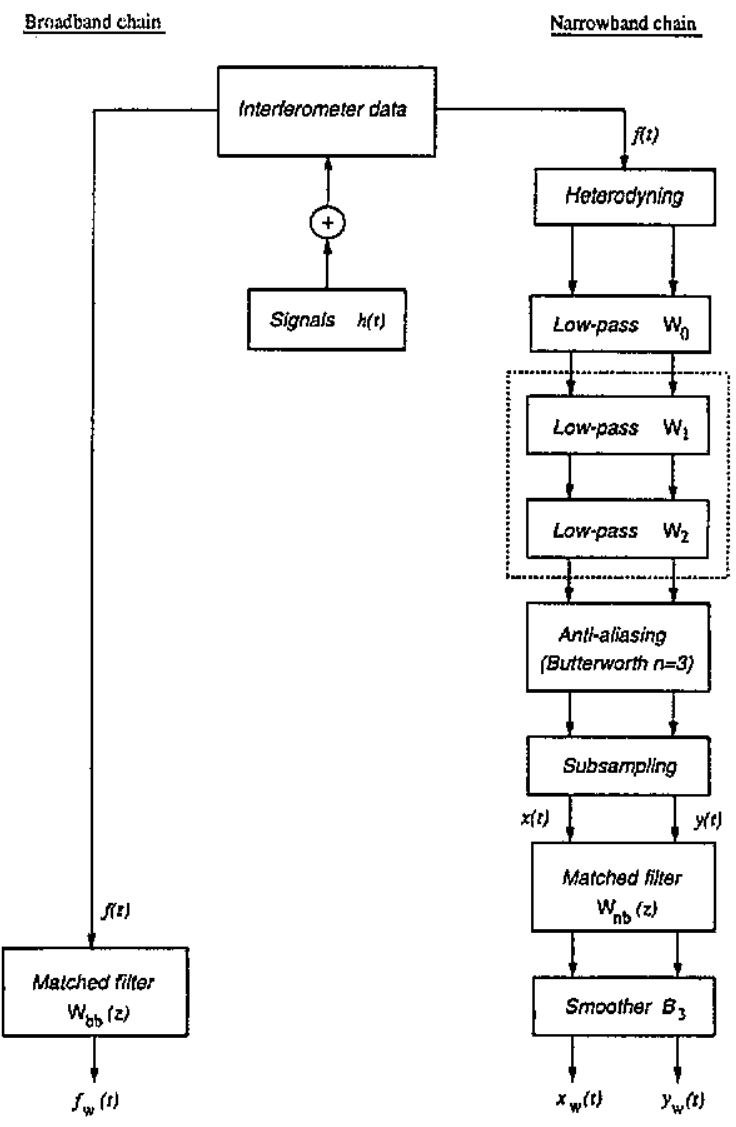

Figure 5. Block diagram of the data analysis chain. Left-hand side: broadband chain; right-hand side: narrowband chain.

to extract the Fourier components around those frequencies.

After heterodyning, the signal becomes complex and its spectrum is shifted so that the original Fourier components around the bar's frequencies are now, respectively, centred at DC. Before letting this complex signal enter the bar model, we filter it with a soft low-pass filter to help clear the data from any excess seismic noise. Immediately after this come the bar filters-a very narrow low-pass (characteristic frequency $\beta_{1}$ ) and an output low-pass $\left(\beta_{2}\right)$.

Up to this point the sampling time has been kept equal to the one originally in the datae.g. $\Delta t_{\text {interf }}=0.1 \mathrm{~ms}$. We must now resample these data in order to match the actual bar's rate, $\Delta t_{\mathrm{bar}}$. But before doing this we insert an anti-aliasing filter to avoid undesired fold-in effects from higher frequencies in the resampled subset. Such a filter can, for example, be implemented as a third-order Butterworth filter, which will be practically flat over the final Nyquist band $1 / \Delta t_{\text {bar. }}$. The output of this filter can be resampled straightaway, i.e. only one data point every $\left(\Delta t_{\mathrm{bar}} / \Delta t_{\mathrm{interf}}\right)$ is retained.

The reduced set obtained by the above procedure can be compared in a coincidence analysis with the data produced by a bar having the same characteristics of this filtering chain. If, for example, one were to look for coincident detection of short bursts of GWs, a suitable filter should be constructed and applied to the processed data to produce a list 
of events on the basis of, say, a threshold-crossing criterion. Such a filter will consist of two stages: a matched filter proper and a smoothing filter $\beta_{3}-\mathrm{cf}$ section 2 abovenecessary to also match the actual bar bandwidth. Coincidences should thereafter be sought between this list and the corresponding one produced from the bar data on the same criteria.

\section{I. A prototype example}

We have implemented the above procedure on a small stretch of real interferometer data drawn from the $100 \mathrm{~h}$ run Garching set. Robust and flexible software has been written for the purpose, which can be considered a prototype for future analyses involving much larger amounts of data.

For the sake of concreteness, we have only considered one of the two bar's resonance frequencies, in this case $925 \mathrm{~Hz}$, and the bandwidth $2 \beta_{3} / 2 \pi$ is that expected for the NAUTILUS antenna. All the parameters of the analysis are shown in table 1.

Table 1.

\begin{tabular}{ll}
\hline Total time analysed & $T_{\text {abs }}=52.4 \mathrm{~s}$ \\
Interferometer sampling time & $\Delta t_{\text {Garching }}=0.1$ \\
Heterodyne frequency & $f_{0}=925 \mathrm{~Hz}$ \\
Heterodyne low-pass filter & $\beta_{0}=312.5 \mathrm{rad} \mathrm{s}^{-1}$ \\
Bar's first low-pass filter & $\beta_{1}=0.01 \mathrm{rad} \mathrm{s}$ \\
Bar's second low-pass filter & $\beta_{2}=156.25 \mathrm{rad} \mathrm{s}$ \\
Antialiasing 3-pole Butterworth & $f_{3-\mathrm{dB}}$ cut-off $=156.25 \mathrm{~Hz}$ \\
Output data sampling time & $\Delta t_{\mathrm{bar}}=6.4 \mathrm{~ms}$ \\
Overall bandwidth & $2 \beta_{3} / 2 \pi=25 \mathrm{~Hz}$ \\
\hline
\end{tabular}

A simple signal model of a broadband burst of gravitational radiation is provided by

$$
h(t)=A \frac{\sin \gamma\left(t-t_{0}\right)}{\gamma\left(t-t_{0}\right)}
$$

where $A$ is the peak amplitude. The signal's peak occurs at time $t_{0}$, and its effective duration is $2 \pi / \gamma$. The spectrum (Fourier transform) of $h(t)$ has the constant value $\pi A / \gamma$ for all frequencies up to $\gamma / 2 \pi$. Note that if we let $\gamma$ go to infinity while the ratio $A / \gamma$ remains constant, the signal turns into a standard $\delta$-function in time.

It is trivial that no physical instrument has an infinite observation bandwidth. This simple observation has the following remarkable consequence: if one such instrument (the interferometer, say) has a bandwidth $B$, it will not be able to tell a 'real' $\delta$-signal from a signal like (24) with $\gamma=B / 2 \pi$ or, indeed, from any such signals with $\gamma \geqslant B / 2 \pi$ having the common ratio $A / \gamma$. This is a fundamental limitation of that particular apparatus, so the experimenter defines a $\delta$-signal as that signal that lasts less than all the time constants of the apparatus, including the correlation time of the filtered data.

Consider now a different instrument-e.g. a resonant bar. By the same argument, the bar experimenter will be forced to set up a different definition of what a $\delta$-signal means to him: since bars have a narrower bandwidth than interferometers, longer signals with non-zero Fourier components at the bar resonance frequencies will be identified as $\delta \mathrm{s}$ in this case.

The proposed narrowbanding filtering of the interferometer data will remove the aforementioned ambiguities-but there $i s$ a price to pay for it: signal-to-noise ratio will be 
sacrificed. More specifically, narrowbanding increases the energy noise of the interferometer filtered data, i.e. we get a larger slope in a semilog plot of the filtered data energy distribution, which is expected to be exponential. For a given threshold there is a larger number of noise events and therefore this procedure will give useful results only if the signals are sufficiently strong so that we can use larger thresholds.

As a matter of fact, if we apply the matched filter to the broadband data (figure 5 , left) we obtain

$$
(S / N)_{\mathrm{bb}}^{2}=\int_{-1 / 2 \Delta t_{\text {inter! }}}^{+1 / 2 \Delta t_{\text {inter }}} \frac{\left|\tilde{h}_{\mathrm{b}}(f)\right|^{2}}{S_{\mathrm{bb}}(f)} \mathrm{d} f
$$

where $\tilde{h}_{\mathrm{bb}}(f)$ is the spectrum of the burst (constant over the interferometer bandwidth) and $S_{\mathrm{bb}}$ is the broadband noise. If we approximate the latter by a constant, we obtain the estimate

$$
(S / N)_{\mathrm{bb}}^{2} \simeq \frac{\tilde{h}_{\mathrm{b}}^{2}}{S_{\mathrm{bb}} \Delta t_{\text {interf }}} .
$$

If, on the other hand, the matched filter is applied at the end of the narrowband chain (figure 5, right), we obtain

$$
(S / N)_{\mathrm{nb}}^{2}=\int_{-1 / 2 \Delta t_{\mathrm{har}}}^{+1 / 2 \Delta t_{\mathrm{har}}} \frac{\left|\tilde{h}_{\mathrm{ab}}(f)\right|^{2}}{S_{\mathrm{nb}}(f)} \mathrm{d} f .
$$

where now $\tilde{h}_{\mathrm{nb}}$ and $S_{\mathrm{nb}}$ are the signal and noise as seen after the narrowband chain. Note that the frequency integration range is now the one suited to our resampling rate. Since both signal and noise are passed through the same chain of filters, we have

$$
\begin{aligned}
& \tilde{h}_{\mathrm{nb}}(f)=W(f) \tilde{h}_{\mathrm{b}}(f) \\
& S_{\mathrm{nb}}(f)=|W(f)|^{2} S_{\mathrm{bb}}(f)
\end{aligned}
$$

where $W(f)$ stands for the global transfer function of the narrowbanding chain. We thus find

$$
(S / N)_{\mathrm{ab}}^{2}=\int_{-1 / 2 \Delta b_{\mathrm{bar}}}^{+1 / 2 \Delta t_{\mathrm{bar}}} \frac{\left|\tilde{h}_{\mathrm{b}}(f)\right|^{2}}{S_{\mathrm{bb}}(f)} \mathrm{d} f
$$

so that, in the same approximation we made earlier

$$
(S / N)_{\mathrm{ab}}^{2} \simeq \frac{\tilde{h}_{\mathrm{b}}^{2}}{S_{\mathrm{bb}} \Delta t_{\mathrm{bar}}} .
$$

Therefore we find that

$$
\frac{(S / N)_{\mathrm{nb}}}{(S / N)_{\mathrm{bb}}} \simeq\left(\frac{\Delta t_{\text {interf }}}{\Delta t_{\mathrm{bar}}}\right)^{1 / 2}=(\text { resampling factor })^{-1 / 2} \text {. }
$$

We still have to consider the effect of the final smoothing filter $\beta_{3}$ : it will always result in a further degradation in signal-noise-ratio, of course. This, however, will not have a dramatic effect whenever the Nyquist band of the resampled data is well matched to $\beta_{3}$. If, on the other hand, it is not, it can easily be shown that there is still a degradation of the order of $\left(\beta_{3} \Delta t_{\text {bar }}\right)$ if $\beta_{3} \ll 1 / \Delta t_{\text {bar }}$, so that a softer resampling factor after narrowbanding cannot offer a way out from losing $(S / N)$ in the process.

We now assess how this works with our prototype model analysis. To this end we have artificially added signals like (24) to the interferometer data. The signals are unusually intense, but this will not invalidate our analysis because we are only looking for ratios in SNR values, and the entire process is linear in the data. 


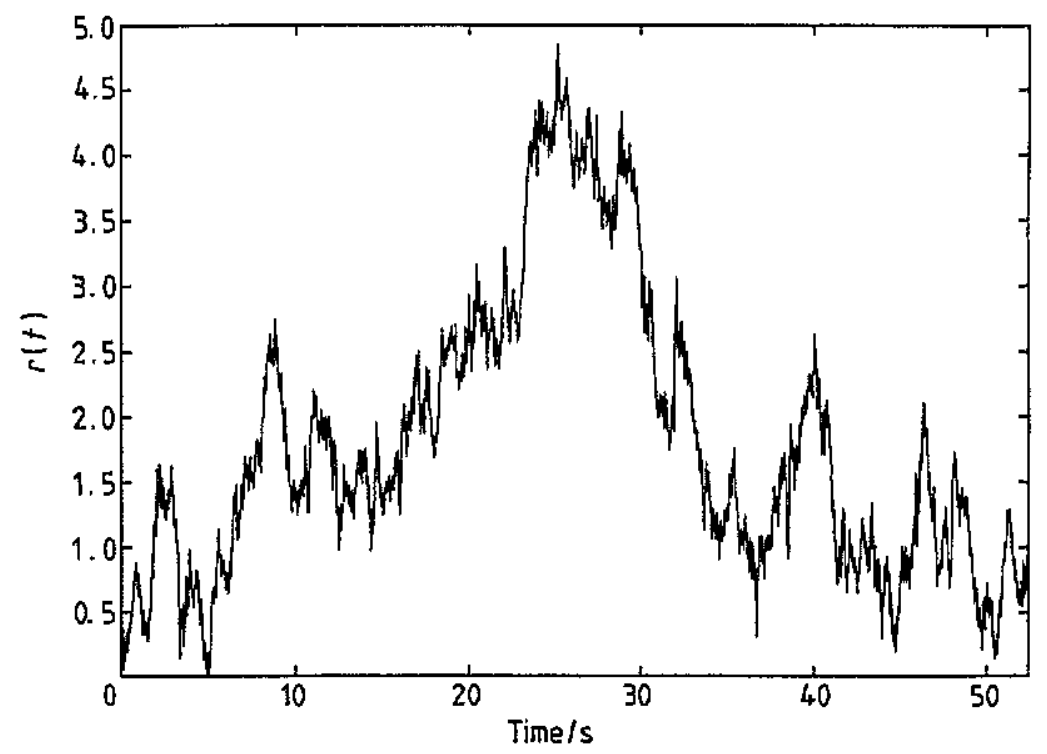

Figure 6. Narrowband chain output. The input is given by the Garching data plus one signal, added at $31.9 \mathrm{~s}$, with parameters: $A=2.0 \times 10^{-15}$, width $=0.2 \mathrm{~ms}$. The quantity shown in ordinates is $r(t)=\left[x^{2}(t)+y^{2}(t)\right]^{1 / 2}$.

For example, a signal with

$$
A=2 \times 10^{-15} \quad \frac{2 \pi}{\gamma}=0.2 \mathrm{~ms}
$$

i.e. a ' $\delta$-for-the-interferometer', is seen with a signal-to-noise ratio of

$$
S / N=5.8 \quad \text { (narrowbanded) }
$$

at the output of a matched filter applied to the narrowbanded interferometer data-cf figure 5 , bottom right. The same signal, if looked for with the appropriate matched filter directly in the interferometer data (figure 5, left) is instead seen with a larger SNR:

$$
S / N=51.9 \quad \text { (broadband). }
$$

Figures 7 and 8 show the outputs of these filters. Figure 6 is included to show the result of narrowbanding the interferometer data with the above signal added at $t_{0}=31.9 \mathrm{~s}$-note that it cannot be seen above the background noise at all at this stage.

We note that for evaluating the SNR against the noise background, we have added several signals, with the same amplitude and width parameters, and then we have evaluated the average of the individual SNRs.

We thus see that this method cannot avoid a degradation in SNR performance. The degradation factor is rather accurately given by $\left(\Delta t_{\text {bar }} / \Delta t_{\text {interf }}\right)^{1 / 2}$, where $\Delta t_{\text {interf }}$ and $\Delta t_{\text {bar }}$ are the sampling times of the interferometer and the bar, respectively. In the case under consideration we have $\Delta t_{\text {interf }}=10^{-4} \mathrm{~s}$ and $\Delta t_{\mathrm{bar}}=6.4 \mathrm{~ms}$, so that $\left(\Delta \mathrm{l}_{\mathrm{bar}} / \Delta t_{\text {interf }}\right)^{1 / 2}=8$, in very good agreement with the observed value 51.9/5.8 $=8.9$.

Now let us see what happens if we add a signal with

$$
A=0.4 \times 10^{-15} \quad \frac{2 \pi}{\gamma}=1.0 \mathrm{~ms}
$$




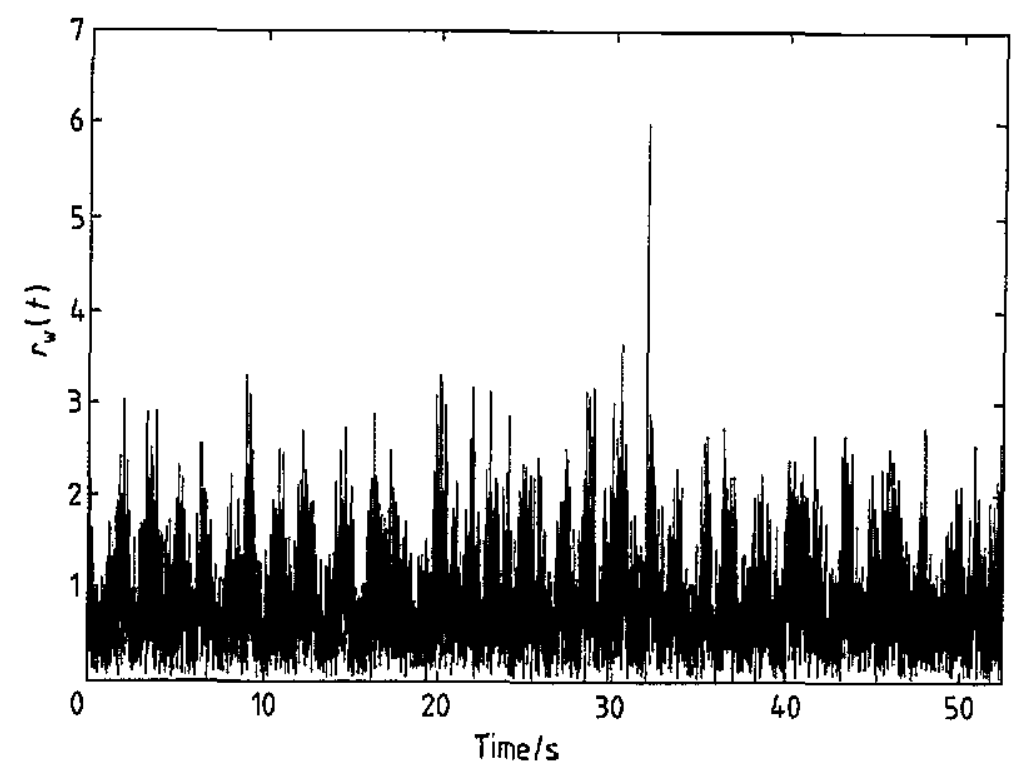

Figure 7. Narrowband chain: $\delta$-matched filter output. The filter is applied to the data shown in figure 6. The variable shown in ordinates is $r(t)=\left[x_{\mathrm{w}}^{2}(t)+y_{\mathrm{w}}^{2}(t)\right]^{1 / 2}$. The SNR for this signal is $(\mathrm{SNR})_{\mathrm{nb}} \simeq 5.8$.

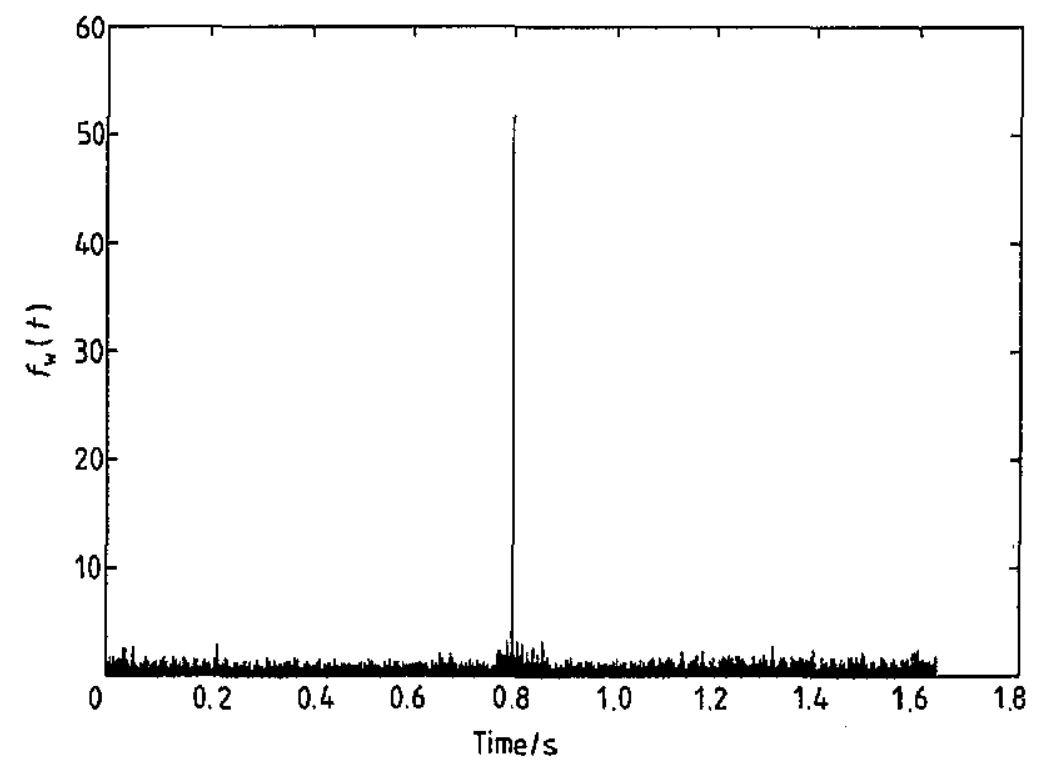

Figure 8. Broadband chain: $\delta$-matched filter output. The input sequence is given by the Garching data plus the same signal. SNR is $(\mathrm{SNR})_{b \mathrm{~b}} \simeq 52$.

This signal is still considered to be a $\delta$ by the bar, but no longer so by the interferometer. Now, since it has the same ratio $A / \gamma$ as in (33), the SNR for its detection after the narrowbanding chain and the same matched filter as before, is also the same as before (we actually obtain a value of 6.3). However, when a matched 


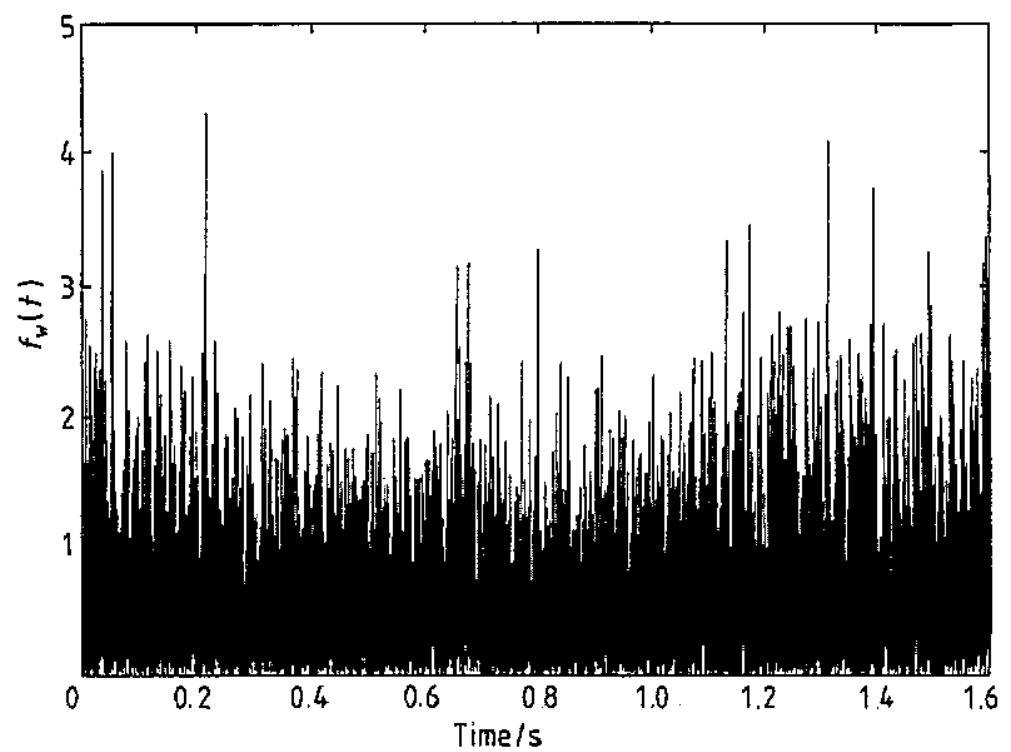

Figure 9. The same as above, except that the signal has now $A=0.4 \times 10^{-15}$, width $=1 \mathrm{~ms}$. SNR is degraded to (SNR)bb $\simeq 3$.

filter suited for $\delta$-detection is applied to the broadband data (figure 5, left) with this signal in them, the SNR is strongly damped $(S N R \simeq 3$ ). Figure 9 shows this situation.

The reason for this is the behaviour of the matched filter impulse response, shown in figure 10: the weights of the broadband matched filter are such that the response to a longer signal is smaller than that to a short signal, that is to a broadband burst, of the same amplitude.

Thus, we conclude that if the two chains, the wideband and the narrowband ones, are both adapted for the search of, say, $1 \mathrm{~ms}$ bursts they may give the same result only if each burst is just $1 \mathrm{~ms}$ long. Otherwise, if different signals are present in the data, then the two chains may give very different results, even opposite, depending on the spectral content of the signal itself.

\section{Conclusion}

Outright standard coincidence analysis between the data produced by a resonant bar detector of gravitational waves and an interferometric detector has a number of problems due to the very different sensitivity characteristics these detectors have. The principal difference is the bandwidth: the interferometer is broadband while the bar is narrowband. In this paper we have considered various types of GW signals: broadband, continuous and stochastic. For broadband bursts we have found that it is always interesting to do the analysis using the full bandwidth of both the detectors, but, in certain cases, by narrowbanding the interferometer data we will have less ambiguous information on the signals in coincidence between bars and interferometers.

For the continuous waves, if in the bandwidth of the bar, the narrowbanding is obviously necessary, and if the interferometer and the bar have comparable broadband burst sensitivity then the bar is more sensitive. 


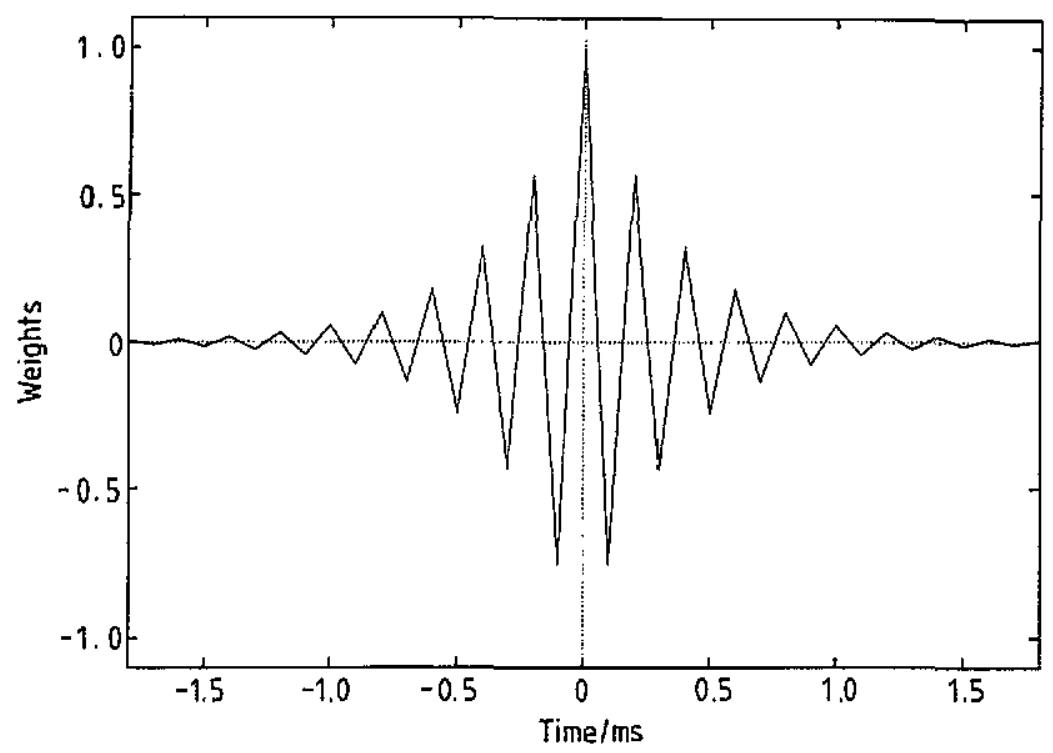

Figure 10. Matched filter impulse response. The weights of the filter are such that the response to a longer signal is smaller than that to a shorter signal (broadband burst) of the same amplitude.

Finally for stochastic waves narrowbanding the interferometer data is absolutely necessary. In certain cases the crosscorrelation of an interferometer output with that of a bar may give better results than cross-correlating two interferometers.

Coincidence is obviously best performed with no time window making allowance for possible time lags between different detectors. An obvious way to realize this is to have the antennæ installed on the same site. Decision making on the issue of building a new detector is a complex matter, but a case can certainly be made for preferential construction at places where there already is a GW observatory. This applies in particular to the new generation of very long baseline interferometers and the newly planned resonant spherical antennæ alluded to in the introduction.

\section{Acknowledgments}

We are grateful to the Garching group for permission to use their data in our simulations in this paper. Two of us (PA and JAL) would like to express gratitude to the Department of Physics and Astronomy in Cardiff for their kind hospitality during the confection of this work. JAL is also grateful to SERC and the Spanish Misnistry of Education for financial support (through a grant and contract PB90-0482-C02-03). We also thank S Frasca, G V Pallottino and G Pizzella for discussions and valuable suggestions.

\section{References}

Abramovici A et al 1992 LIGo: the laser interferometer gravitational wave observatory Science 256 325-33 Astone P et al 1993 Phys. Rev. D 472

Astone, P, Bonifazi P and Pallottino G V 1991 Rev. Sci. Instrum. 613899

Blair D (ed) 1991 The Detection of Gravitational Waves (Cambridge: Cambridge University Press)

Bonifazi P, Frasca S, Pallottino G V, Pizzella G and Ferrari V 1978 Nuovo Cimento C 1465 
Bradaschia C et al 1990 The Virgo project-a wide band antenna for gravitational-wave detection Nucl. Instrum. Meth. Phys. A 289 518-25

Compton K, Nicholson D and Schutz B F 1993 in preparation

Drever R W P 1983 Gravitational Radiation ed N Deruelle and T Piran (Amsterdam: North-Holland) pp 321-38

Finn S and Chernoff D F 1993 Phys. Rev. D 472198

Giazotto A 1989 Phys. Rep. 182 365-425

Lobo J A 1992 Class. Quantum Grav. 91385

Mackenzie N L, Ward H, Hough J, Kerr G A, Mangan J B, Meers B J, Newton G P, Robertson D I and Robertson N 1989 Data acquisition and analysis with the Glasgow prototype detector Gravitational Wave Data Analysis ed B F Schutz (Dordrecht: Kluwer) pp 269-74

Maischberger K, Ruediger A, Schilling R, Schnupp L, Winkler W and Leuchs G 1991 Status of the Garching 30 meter prototype for a large gravitational wave detector ed P F Michelson, En-Ke Hu and G Pizzella Experimental Gravisational Physics (Singapore: World Scientific) pp 316-21

Meers B J 1988 Recycling in laser-interferometric gravitational-wave detectors Phys. Rev. D 38 2317-326

Michelson P F 1987 On detecting stochastic background gravitational radiation with terrestrial detectors Mon. Not. R. Astron. Soc. $227933-41$

Michelson P F, Hu En-Ke and Pizzella G (ed) 1991 Experimental Gravitational Physics (Singapore: World Scientific)

Niebauer T M, Ruediger A, Schilling R, Schnupp L, Winkler W and Danzmann K 1993 Phys, Rev. D 473106

Pallottino G V and Pizzella G 1984 Nuovo Cimento C 7155

Schutz B F 1991 Data processing anajysis and storage for interferometric antennas The Detection of Gravitational Waves ed D Blair (Cambridge: Cambridge University Press)

Schutz B F (ed) 1989 Gravitational Wave Data Analysis (Dordrecht: Kluwer)

Ward H, Hough J, Kerr G A, Mackenzie N L, Mangan J B, Meers B J, Newton G P, Robertson D I and Robertson N A 1991 The Glasgow gravitational wave detector-present progress and future plans Experimental Gravitational Physics ed P F Michelson, En-Ke Hu and G Pizzella (Singapore: World Scientific) pp 322-7

Watkins W J 1991 PhD thesis University of Wales College of Cardiff 